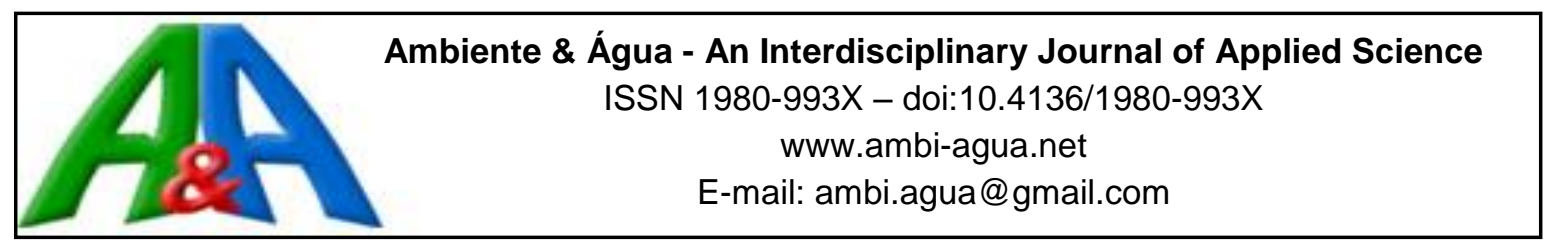

\title{
Cálculo de precipitação média utilizando método de Thiessen e as linhas de cumeada
}

\author{
ARTICLES doi:10.4136/ambi-agua.1906
}

Received: 31 Mar. 2016; Accepted: 08 Dec. 2017

\author{
Alexandre Germano Marciano*; Alexandre Augusto Barbosa; Ana Paula Moni Silva \\ Universidade Federal de Itajubá (UNIFEI), Itajubá, MG, Brasil \\ Instituto de Recursos Naturais (IRN).E-mail: agermano@unifei.edu.br, \\ barbosa@unifei.edu.br, anamoni@unifei.edu.br \\ *Autor correspondente
}

\section{RESUMO}

O presente estudo propõe um método para calcular as áreas de influência baseado no Método de Thiessen e considerando as linhas de cumeada do relevo. O estudo foi estabelecido para a bacia do Alto Sapucaí, a montante da cidade de Itajubá-MG, e foram usados os dados das estações de monitoramento de enchentes da Universidade Federal de Itajubá. Também foram utilizadas imagens da Shuttle Radar Topography Mission (SRTM). Com esses dados, foram traçadas áreas de influência das estações pluviométricas, por meio da junção do Método de Thiessen e das linhas de cumeadas mais próximas dessas medições de pluviosidade. Acredita-se que com isso os resultados das áreas de influência de uma estação climatológica sejam mais precisos que outros métodos recomendados pela bibliografia especializada.

Palavras-chave: bacia hidrográfica, pluviometria, polígonos de Thiessen.

\section{Calculation of medium precipitation using the Thiessen method and the ridge}

\begin{abstract}
This study proposes a method to calculate hydrological areas of influence based on the Thiessen method and considering the ridge line. The study dealt with the basin of the high Sapucai, upstream of the city of Itajubá-MG, and used data from flood monitoring stations of the Federal University of Itajubá as well as images of the Shuttle Radar Topography Mission (SRTM). Through the use of this data, areas of influence were traced of rainfall stations through a combination of the Thiessen method and the lines ridges nearest mountain of these rainfall measurements. It is believed that, using results of the areas of influence of a meteorological station, this method produces results that are more accurate than other methods recommended by the specialized literature.
\end{abstract}

Keywords: pluviometry, Thiessen polygons, watershed. 


\section{INTRODUÇÃO}

Bacia hidrográfica é a área de captação natural da água da precipitação, onde ocorre a drenagem em ravinas, canais e tributários, para um curso de água principal, tendo a vazão uma única saída: desaguar num curso d'água maior, lago ou oceano (Shinzato et al., 2009).

A compreensão da precipitação numa bacia hidrográfica é muito ampla, pois há muitas variáveis de tempo e espaço envolvidas na área estudada. A precipitação dificilmente segue um padrão físico idêntico. A variação espacial muda rapidamente, pois o núcleo de chuva se modifica a todo momento e a variação temporal é extremamente aleatória - chuvas podem ser de alguns minutos até várias horas ou dias e com uma grande amplitude de intensidade.

Numa bacia hidrográfica é muito difícil saber o volume exato de chuva para toda a bacia, mas esse valor é indispensável para os estudos hidrológicos. Segundo Pedrazzi (1999), há 3 métodos para o cálculo da chuva média: método da média aritmética, método de Thiessen e método das isoietas. Dentre esses métodos o mais utilizado é o de Thiessen. O da média aritmética é muito simplório - por não levar em conta a distribuição de estações pluviométricas e o relevo; o método das isoietas é mais complexo de ser implementado pela necessidade de uma grade de informações pontuais de precipitação para a área estudada.

Diversos autores utilizaram (e utilizam) o Método de Thiessen (1911) para aplicações em recursos hídricos e previsão climática obtendo resultados satisfatórios. Tal método foi desenvolvido pelo meteorologista americano Alfred $\mathrm{H}$. Thiessen, que aplicou a teoria do diagrama de Voronoi para o cálculo da precipitação média na região de estudo.

A topografia influencia o movimento do ar, tendo assim um efeito sobre o clima, por exemplo as cadeias de montanhas, que são barreiras que alteram os padrões de vento e precipitação (Funhen, 2015). De acordo com Carvalho e Assad (2005), os maiores valores de precipitação tendem a se concentrar nas maiores altitudes, devido aos ventos úmidos orográficos que porventura se estabelecem em cadeia de montanhas, as quais servem de barreiras naturais. Essa precipitação adicional (em relação a uma área plana) depende das dimensões e alinhamentos dessas elevações em relação aos ventos orográficos, bem como da umidade da parcela de ar e das condições atmosféricas. Ademais, as vertentes a barlavento recebem mais precipitação do que as vertentes a sota-vento, devido aos efeitos de expansão e compreensão adiabática dos ventos úmidos (Ayoade, 1996; Barry e Chorley, 2010).

Com os dados de precipitação gerados no Sistema de Monitoramento de Enchentes do Laboratório de Informações Hídricas da Universidade Federal de Itajubá, com o emprego do Método de Thiessen e com a inserção das montanhas como barreiras naturais, propõe-se neste trabalho a obtenção das áreas de influência de cada estação pluviométrica. Dessa maneira, em toda essa área de influência haverá uma precipitação média que servirá como base para qualquer trabalho com estabelecimento de escoamentos superficiais, evaporação, infiltração e escoamento subterrâneo.

\section{MATERIAL E MÉTODOS}

A bacia hidrográfica do rio Sapucaí integra a bacia do rio Grande, e esta, por sua vez, faz parte da bacia do Plata, rio que desemboca no oceano Atlântico. A bacia do rio Sapucaí está localizada na região Sudeste do Brasil atravessando os Estados de São Paulo, com $32 \mathrm{~km}$ de extensão e de Minas Gerais, com 308 km. O rio Sapucaí nasce na Serra da Mantiqueira, no município de Campos do Jordão - SP, na cota de 1680 metros e deságua no reservatório de Furnas, na cota 760 metros.

Algumas cidades ao longo do rio Sapucaí sofrem com eventos recorrentes de cheias, como por exemplo, os municípios de Itajubá, Piranguinho, Santa Rita do Sapucaí e Pouso Alegre. A 
cidade de Itajubá está localizada entre o alto e o médio Sapucaí. Situada no Sul do Estado de Minas Gerais, conta com uma área de 294 km² e uma população em torno de 95.000 habitantes, dos quais aproximadamente $91 \%$ residem na área urbana (IBGE, 2014). As cheias existem desde sua fundação e é algo que tem preocupado as autoridades e a população, esta tem sofrido grandes prejuízos com os eventos extremos de cheias ao longo dos anos (Pinheiro, 2005). Neste caso, o motivo destas cheias, segundo Moni Silva (2006), é que as planícies inundáveis apresentam uma topografia favorável às ocupações humanas, com seus terrenos planos, sendo praticamente a única área passível de desenvolvimento, pois prevalece o domínio de vales encaixados e estreitos da Serra da Mantiqueira.

No trabalho de Barbosa et al. (2015), foi proposto um sistema de monitoramento de cheias para a bacia do alto do Sapucaí, no município de Itajubá/MG, onde foram utilizados: sensores de pressão instalados dentro do rio, os quais serviam para medir a variação das colunas d'água; pluviômetros que mediam a intensidade e quantidade da precipitação; uma placa fotovoltaica que fornecia energia ao sistema; e um transmissor via telefonia celular que transmitia os dados coletados via SMS (Short Message Service). Os objetivos desse sistema eram gerar informações de natureza, intensidade e duração dos eventos de cheias à comunidade, além de soluções não usuais para a problemática das enchentes. A partir de 2009, através do Laboratório de Informações Hídricas da Universidade Federal de Itajubá - UNIFEI, foram instaladas 18 estações de monitoramento de cheias, que beneficiavam as cidades da região: Itajubá, Piranguinho, Pouso Alegre, Santa Rita do Sapucaí, Delfim Moreira, Maria da Fé, Brazópolis e Piranguçu. O Sistema foi desativado em janeiro de 2015, sendo mantidas 6 estações para pesquisas acadêmicas.

Para determinar as áreas de influência dos pluviômetros das estações será usado o Método de Thiessen. Este Método gera uma média ponderada das alturas registradas pelos pluviômetros, que é diretamente proporcional à área de influência da bacia, considera a não uniformidade da distribuição espacial das estações, e não leva em conta o relevo da bacia (Tucci, 1997). Conforme afirma Porto et al. (2011), os resultados são consideráveis em terrenos levemente acidentados, quando a localização e exposição dos pluviômetros são semelhantes, e as distâncias entre eles não são muito grandes.

O Método de Thiessen (1911) consiste em:

a) unir as estações por trechos retilíneos;

b) traçar linhas perpendiculares aos trechos retilíneos sobre a mediatriz da linha que liga as estações;

c) alongar as linhas perpendiculares até encontrar outra mediatriz.

O polígono de Thiessen é formado pela interseção das linhas das mediatrizes, correspondendo à área de influência de cada estação.

A área de influência possui um peso perante a área total, expresso pela Equação 1:

$W i=\frac{A i}{A}$

Onde:

Wi é o fator de peso;

$A i$ é a área de influência da estação;

$A$ é a área total da bacia hidrográfica.

A precipitação média é expressa na Equação 2:

$P_{m}=\frac{\sum A_{i} P_{i}}{A}$ 
Onde:

$P_{m}$ é a precipitação média na bacia $(\mathrm{mm})$

$P_{i}$ é a precipitação na estação $(\mathrm{mm})$

$A_{i}$ é a área de influência da estação;

$A$ é a área total da bacia.

Os polígonos de Thiessen foram traçados na bacia do Rio Sapucaí, conforme distribuição das estações (Figura 1).

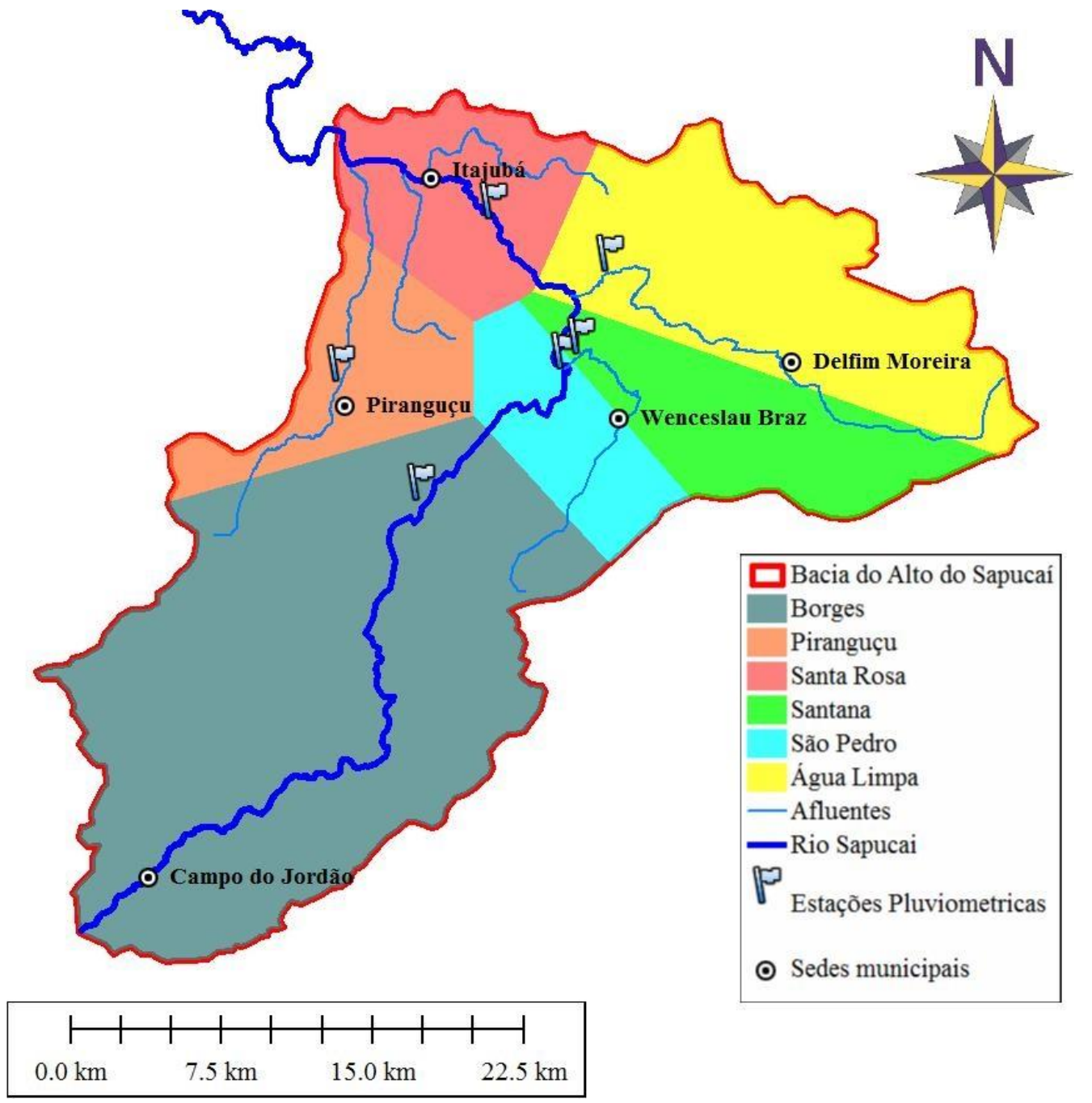

Figura 1. Polígono de Thiessen na bacia do rio Sapucaí-MG.

Para análise da topografia, a NASA disponibiliza gratuitamente um modelo digital de elevação, que são os dados da Shuttle Radar Topography Mission (SRTM), que é uma rede de células quadradas de $90 \mathrm{~m}$ de lado contendo valores acurados de altimetria.

No domínio do modelo digital de elevação da bacia analisada, foram determinadas as linhas de cumeadas. Para isso, foi utilizado o módulo Grid do Arc/INFO, versão 10.2.

Em topografia, linha de cumeada é a linha formada pelos pontos mais altos da montanha, no sentido longitudinal (Guimarães et al., 2011). Tendo a altitude como critério de definição da linha de cumeada, essas linhas são cristas prolongadas, vislumbradas no relevo. As linhas de cumeada obtidas para a região de estudo são mostradas na Figura 2. 


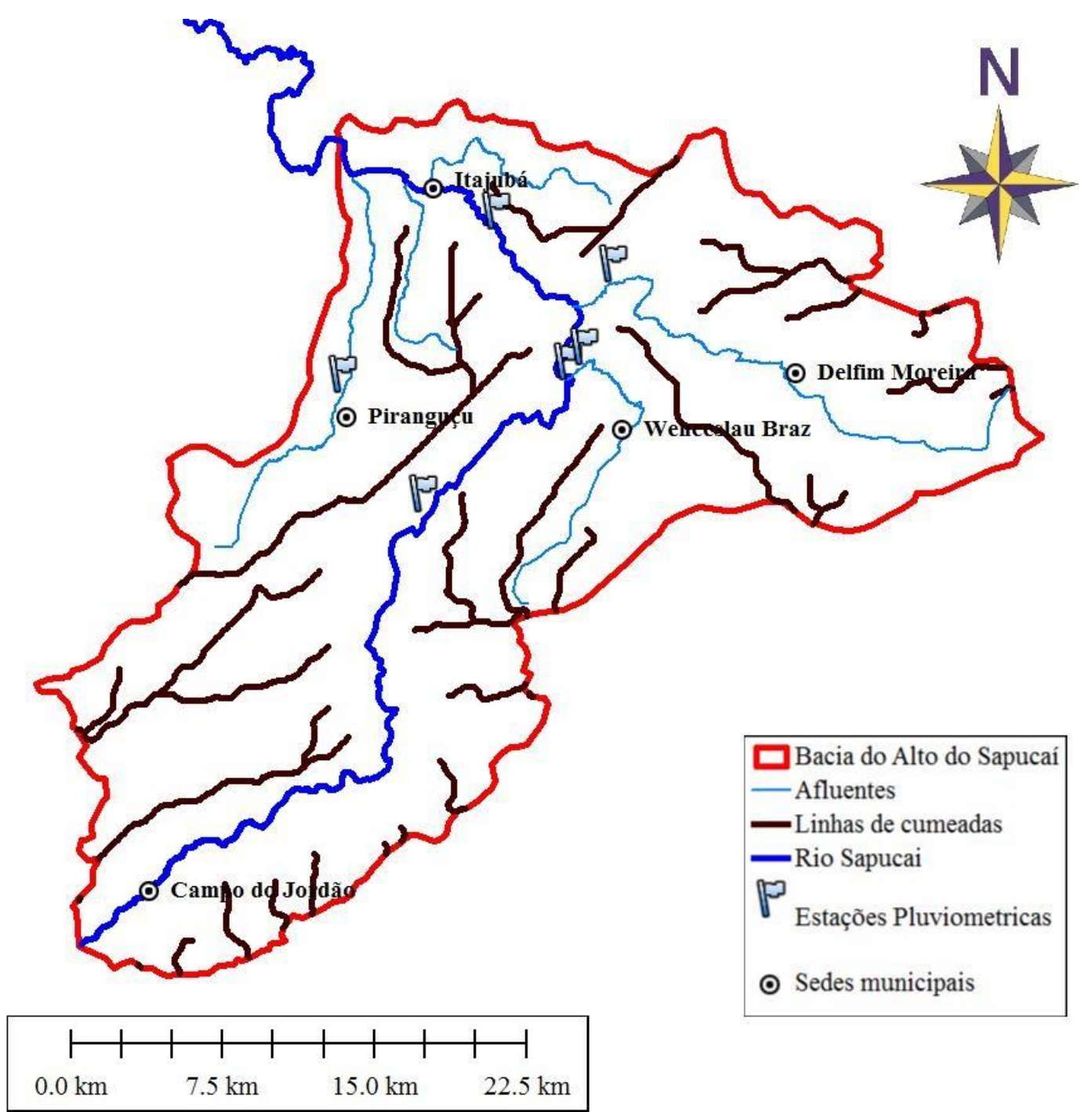

Figura 2. Linhas de cumeadas da bacia do rio Sapucaí-MG.

A junção dos polígonos de Thiessen e das linhas de cumeada foram realizadas com o uso do programa ArcViewGis 10.2.

\section{RESULTADOS}

De acordo com o exposto sobre o uso do Método de Thiessen e o uso das linhas de cumeadas como barreiras naturais na bacia do rio Sapucaí, intenta-se a partir desses métodos criar uma área que tenha a influência do polígono de Thiessen e também a influência das barreiras naturais.

As novas áreas de influência de cada uma das estações de monitoramento estão mostradas na Figura 3.

\section{IPABH}




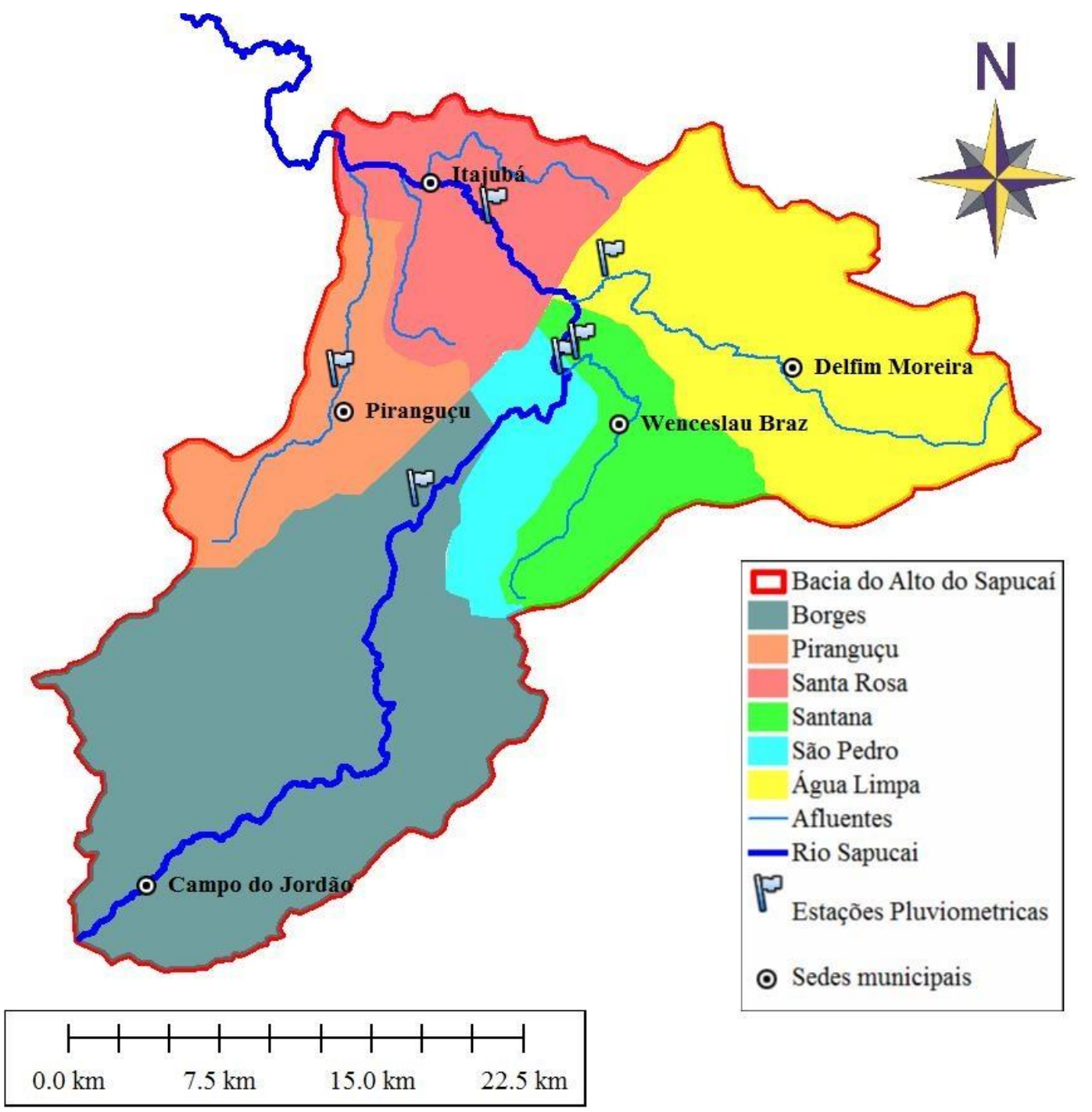

Figura 3. Áreas de influência pelo método proposto.

Na Tabela 1 são apresentados valores das grandezas calculadas do método de Thiessen e do método proposto. 
Tabela 1. Valores calculados do Método de Thiessen e do Método Proposto aplicado à bacia do alto Sapucaí.

\begin{tabular}{|c|c|c|c|c|c|c|c|c|}
\hline \multirow{2}{*}{ Estação } & \multicolumn{2}{|l|}{ Área } & \multicolumn{5}{|c|}{ Precipitação (mm) } & \multirow{2}{*}{$\frac{\text { Ponderação }}{P i^{*} W i(\mathrm{~mm})}$} \\
\hline & Área $\left(\mathbf{k m}^{2}\right)$ & $W i(1)$ & 2011 & 2012 & 2013 & 2014 & Média (2) & \\
\hline \multicolumn{9}{|c|}{ Método Thiessen } \\
\hline Borges & 444,07 & 0,46 & 1644,0 & 1794,2 & 1710,8 & 818,2 & 1491,8 & 680,7 \\
\hline São Pedro & 65,02 & $\mathbf{0 , 0 7}$ & 1397,8 & 1385,8 & 1397,0 & 828,0 & 1252,1 & 83,7 \\
\hline Santana & 104,75 & 0,11 & 1377,2 & 1275,2 & 1298,8 & 710,4 & 1165,4 & 125,4 \\
\hline Água Limpa & 182,74 & 0,19 & 1156,6 & 1408,4 & 1339,6 & 924,4 & 1207,2 & 226,7 \\
\hline Santa Rosa & 94,75 & 0,10 & 1133,8 & 1068,6 & 1415,0 & 765,4 & 1095,7 & 106,7 \\
\hline Piranguçu & 81,95 & $\mathbf{0 , 0 8}$ & 1263,3 & 1544,5 & 1672,8 & 818,3 & 1324,7 & 111,5 \\
\hline \multirow{2}{*}{\multicolumn{9}{|c|}{ Precipitação média da Área Total }} \\
\hline & & & & & & & & \\
\hline Borges & 379,05 & $\mathbf{0 , 3 9}$ & 1644,0 & 1794,2 & 1710,8 & 818,2 & 1491,8 & 581,0 \\
\hline São Pedro & 53,31 & 0,05 & 1397,8 & 1385,8 & 1397,0 & 828,0 & 1252,1 & 68,6 \\
\hline Santana & 86,97 & 0,09 & 1377,2 & 1275,2 & 1298,8 & 710,4 & 1165,4 & 104,1 \\
\hline Água Limpa & 231,72 & 0,24 & 1156,6 & 1408,4 & 1339,6 & 924,4 & 1207,2 & 287,4 \\
\hline Santa Rosa & 125,21 & $\mathbf{0 , 1 3}$ & 1133,8 & 1068,6 & 1415,0 & 765,4 & 1095,7 & 141,0 \\
\hline Piranguçu & 97,02 & $\mathbf{0 , 1 0}$ & 1263,2 & 1544,5 & 1672,7 & 818,2 & 1324,7 & 132,0 \\
\hline Total & 973,28 & $\mathbf{1 , 0 0}$ & \multicolumn{5}{|c|}{ Precipitação média da Área Total } & 1314,1 \\
\hline
\end{tabular}

Na Tabela 2 mostram as comparações entre os 2 métodos empregados neste estudo.

Tabela 2. Comparações entre o Método de Thiessen e o Método Proposto.

\begin{tabular}{lccccc}
\hline \multirow{2}{*}{ Estação } & \multicolumn{2}{c}{ Fator de peso } & \multicolumn{3}{c}{ Ponderação } \\
\cline { 2 - 6 } & Wi Thiessen & Wi Proposto & Pi $^{*}$ Wi Thiessen & Pi*Wi Proposto & Diferença (\%) \\
\hline Borges & 0,46 & 0,39 & 680,7 & 581,0 & -17 \\
São Pedro & 0,07 & 0,05 & 83,7 & 68,6 & -22 \\
Santana & 0,11 & 0,09 & 125,4 & 104,1 & -20 \\
Água Limpa & 0,19 & 0,24 & 226,7 & 287,4 & 21 \\
Santa Rosa & 0,10 & 0,13 & 106,7 & 141,0 & 24 \\
Piranguçu & 0,08 & 0,10 & 111,5 & 132,1 & 16 \\
\hline Total & $\mathbf{1 , 0 0}$ & $\mathbf{1 , 0 0}$ & $\mathbf{1 3 3 4 , 6}$ & $\mathbf{1 3 1 4 , 1}$ & 2 \\
\hline
\end{tabular}

\section{CONCLUSÃO}

Diante das comparações dos métodos, constata-se que houve diferenças nos valores de áreas de influência de todas as estações de medição: algumas tiveram suas áreas aumentadas e outras, diminuídas.

O método proposto emprega o Método de Thiessen o qual usa a área da bacia e a localização das estações para traçar os polígonos e as linhas de cumeadas do relevo, que são geradas a partir de modelo digital de elevação, tornando o método proposto mais laborioso no sentido de ostentar informações de topografia.

O Método de Thiessen não é satisfatório para aplicação em terrenos acidentados. Com isso, o método proposto possivelmente tem uma maior representatividade quanto à precipitação média em uma área, pois as linhas de cumeada das montanhas são divisores de águas e alteram o comportamento das chuvas de ambos os lados das vertentes. 
O cálculo de precipitação média em uma determinada área é usualmente utilizado em estudos de escoamentos superficiais, evaporação, infiltração e escoamento subterrâneo. Assim, uma melhor estimativa desse valor de precipitação média é essencial para que os valores obtidos por meio dela sejam os mais próximos da realidade.

Em fase de estudos estão novas aplicações desse método em outras bacias hidrográficas.

\section{REFERÊNCIAS}

AYOADE, J. O. Introdução à Climatologia para os trópicos. 4. ed. Rio de Janeiro: Bertrand do Brasil, 1996. 159-176 p.

BARBOSA, A. A.; OLIVEIRA, G. M.; OLIVEIRA, T. J. Relatório da Comissão de Avaliação Técnica para Recuperação e Urbanização das Margens do Rio Sapucaí e seus Afluentes da Área Urbana. Revista Meio Ambiente e Sustentabilidade, v. 9, n. 4, p. 126-140, 2015.

BARRY, R. G.; CHORLEY, R. J. Atmosphere, weather, and climate. 9. ed. New York: Routledge, 2010. p. 62-74.

CARVALHO, J. R. P.; ASSAD, E. D. Análise espacial da precipitação pluviométrica no Estado de São Paulo: Comparação de métodos de interpolação. Engenharia Agrícola, v. 25, n. 2, p. 377-384, 2005.

FUNHEN. Como o Movimento Air afetar o clima. Disponível em: http://www.funhen.com/como-o-movimento-air-afetar-o-clima. Acesso em: 22 abr. 2015.

GUIMARÃES, V.; CHIMENES, M. M.; ANDRADE, C.; BEZERRA, R. A. O Novo código florestal e seus reflexos para a gestão ambiental. In: CONGRESSO BRASILEIRO DE GESTÃO AMBIENTAL, 2., 1-7 nov., 2011, Dourados. Anais... Bauru: IBEAS, 2011.

INSTITUTO BRASILEIRO DE GEOGRAFIA E ESTATÍSTICA. Cidades. 2014. Disponível em: http: www.ibge.gov.br. Acesso em: 15 maio 2015.

MONI SILVA, A. P. Elaboração de Manchas de Inundação para o Município de Itajubá, utilizando SIG. 2006. 38f. Dissertação (Mestrado em Engenharia da Energia) Universidade Federal de Itajubá, Itajubá, 2006.

PEDRAZZI, J. A. Precipitação. 1999. Disponível em: http://www.ecivilnet.com/apostilas/files/hidrologiaprecipitacao.zip. Acesso em 01 abr. 2015.

PINHEIRO, V. M. Avaliação Técnica e Histórica das Enchentes em Itajubá - MG. 2005. 30f. Dissertação (Mestrado em Engenharia da Energia) - Universidade Federal de Itajubá, Itajubá, 2005.

PORTO, M. M; ANDRADE, E. M.; TEIXEIRA, A. S. Emprego do Arcview na definição das regiões de precipitações homogêneas para o estado do Ceará. In: SIMPÓSIO DE RECURSOS HÍDRICOS DO CENTRO OESTE, 2., 2002, Campo Grande. Anais... São Paulo: ABAS, 2002. 1 CD-ROM.

SHINZATO, E. T.; GASPARORO, E. A. G.; TONELLO, K. C. Monitoramento da precipitação efetiva em diferentes povoamentos florestais: estudo de caso na Floresta Nacional de Ipanema, Iperó-SP. In: SEMINÁRIO DE RECURSOS HÍDRICOS DA BACIA HIDROGRÁFICA DO PARAÍBA DO SUL: RECUPERAÇÃO DE ÁREAS DEGRADADAS SERVIÇOS AMBIENTAIS E SUSTENTABILIDADE, 2., 09-11. 2009, Taubaté. Anais... Taubaté: UNITAU, 2009. 
THIESSEN, A. H. Precipitation averages for large areas. Monthly Weather Review, v. 39, n.7, p. 1082-1089, 1911.

TUCCI, C. E. M. Hidrologia: ciência e aplicação. 2. ed. Porto Alegre: Editora da Universidade, 1997. $195 \mathrm{p}$. 